\title{
A Strange Star Scenario for the Formation of Eccentric Millisecond Pulsar/Helium White Dwarf Binaries
}

\author{
Long Jiang ${ }^{1}$, Xiang-Dong $\mathrm{Li}^{1,2}$, Jishnu Dey ${ }^{3}$, and Mira Dey ${ }^{3}$ \\ ${ }^{1}$ Department of Astronomy, Nanjing University, Nanjing 210046, China \\ ${ }^{2}$ Key laboratory of Modern Astronomy and Astrophysics (Nanjing University), Ministry of \\ Education, Nanjing210046, China; lixd@nju.edu.cn \\ ${ }^{3}$ Department of Physics, Presidency University, 86/1, College Street, Kolkata 700073, \\ India; jishnu.dey@gmail.com,mira.dey@gmail.com
}

\begin{abstract}
According to the recycling scenario, millisecond pulsars (MSPs) have evolved from low-mass X-ray binaries (LMXBs). Their orbits are expected to be circular due to tidal interactions during the binary evolution, as observed in most of the binary MSPs. There are some peculiar systems that do not fit this picture. Three recent examples are PSRs J2234+06, J1946+3417 and J1950+2414, all of which are MSPs in eccentric orbits but with mass functions compatible with expected He white dwarf companions. It has been suggested these MSPs may have formed from delayed accretion-induced collapse of massive white dwarfs, or the eccentricity may be induced by dynamical interaction between the binary and a circumbinary disk. Assuming that the core density of accreting neutron stars in LMXBs may reach the density of quark deconfinement, which can lead to phase transition from neutron stars to strange quark stars, we show that the resultant MSPs are likely to have an eccentric orbit, due to the sudden loss of the gravitational mass of the neutron star during the transition. The eccentricities can be reproduced with a reasonable estimate of the mass loss. This scenario might also account for the formation of the youngest known X-ray binary Cir $\mathrm{X}-1$, which also possesses a low-field compact star in an eccentric orbit.
\end{abstract}

Subject headings: stars: neutron - X-rays: binaries - pulsars: individual (PSR J2234+06, PSR J1946+3417) 


\section{Introduction}

Millisecond pulsars (MSPs) are low-field $\left(B \sim 10^{8}-10^{9} \mathrm{G}\right)$, rapidly rotating neutron stars (NSs) with spin periods $P_{\mathrm{s}} \lesssim 30 \mathrm{~ms}$ (Lorimer 2008). In the standard recycling scenario (Alpar et al. 1982; Radhakrishnan \& Srinivasan 1982; Bhattacharya \& van den Heuvel 1991), binary MSPs are regarded as the descendants of low-mass X-ray binaries (LMXBs), in which a NS has accreted sufficient mass from its companion star, and spun up to millisecond periods. Recent observations of the LMXB/MSP transition in PSRs J1023+0038 (Archibald et al. 2009), J1824-2452I (Papitto et al. 2013), and J1227-4853 (Roy et al. 2015) have lent strong support to the evolutionary link between LMXBs and MSPs.

If the initial orbital period of a LMXB is longer than the so-called bifurcation period (e.g., Pylyser \& Savonije 1989), the mass transfer begins when the companion star evolves to be a (sub)giant. The binary orbit continues expanding until the donor star loses most of its hydrogen envelope, forming a low-mass He white dwarf (WD). Since the mass transfer timescale is much longer than tidal circularization timescale, the orbits of binary MSPs are highly circularized (Phinney 1992), except those in globular clusters where the orbits may be perturbed by dynamical interactions in the dense stellar environment.

The discovery of PSR J1903+0327 presents a challenge to the recycling scenario, which is a binary MSP with a high eccentricity $(e \sim 0.44)$ (Champion et al. 2008). Located in the Galactic field, this pulsar is accompanied by a G-type main-sequence secondary. It was suggested to stem from a hierarchical triple system (Freire et al. 2011; Portegies Zwart et al. 2011; Pijloo et al. 2012), or be a newborn NS experienced supernova fallback disk accretion (Liu \& Li 2009). Since then, some more eccentric MSPs have been discovered, including PSRs J2234+06 (Deneva et al. 2013), J1946+3417 (Barr er al. 2013) and J1950+2414 (Knispel et al. 2015)1. All of these objects are Galactic field MSPs with a He WD (of mass around $0.24 M_{\odot}$ for a $1.4 M_{\odot} \mathrm{NS}$ ) in orbits with periods ranging from 22 to 32 days. The short spin periods ( $2 \lesssim P_{\mathrm{s}} \lesssim 12 \mathrm{~ms}$ ) indicate that they have experienced extensive mass transfer processes during the LMXB phase, but the eccentric orbits (with $0.027<e<0.13$ ) are difficult to understand in standard LMXB evolution scenarios. Like in the case of PSR $\mathrm{J} 1903+0327$, it is still in principle possible that all these systems formed by the disruption of a triple system. However, the strong similarity in the orbital properties of all the recently discovered pulsars is not what one would expect from the disruption of a triple system: this is a chaotic process that should produce a wide range of orbital periods and orbital

\footnotetext{
${ }^{1}$ Another MSP with an eccentric orbit will be presented in Camilo et al. (2015, in preparation). A previously known system, PSR J1618-3919 might also be related: it has a spin period of $12 \mathrm{~ms}$ and an orbital eccentricity of 0.027 (Bailes 2010).
} 
eccentricities. The close similarity of these systems suggests instead a common evolutionary mechanism, different from what formed PSR J1903+0327.

Freire \& Tauris (2014) suggested that these MSPs formed from delayed accretion-induced collapse of massive WDs. According to their scenario, the progenitor binary may consist of a $7 M_{\odot}$ primary and a $2 M_{\odot}$ secondary. The primary star evolves to be an ONeMg WD of mass $1.2 M_{\odot}$ while the secondary is still on its main sequence stage. In the following evolution, the secondary fills its Roche lobe and transfers hydrogen-rich material to the WD, so that the mass of WD increases to the Chandrasekhar limit. However, if the WD is fast spinning, it may keep accreting without collapsing to be a NS (e.g. Yoon \& Langer 2004). The WD-NS conversion only occurs when the WD has spun down and the centrifugal forces that sustain the star are weakened, which might take several Gyr. Then the sudden released gravitational binding energy during the collapse increases the orbital period and imposes an eccentricity to the former circularized orbit. The re-circularization times for such MSP/WD binaries are generally much longer than Hubble time (Zahn 1977; Hut 1981). According to the calculation by Tauris et al. (2013), there is an optimal range of orbital periods where the mass transfer is both dynamically stable (i.e., without entering common envelope evolution) and rapid enough for stable thermonuclear burning of Hydrogen (e.g., Yaron et al. 2005). This range of orbital periods goes from 10 to 60 days, and the middle of this range is right where the aforementioned systems are.

Alternatively, Antoniadis (2014) proposed that the eccentricities of the two MSPs may be caused by dynamical interaction between the binary and a circumbinary disk, which formed from the material escaping the donor star during hydrogen-shell flashes shortly before the WD cooling phase. Adopting a linear perturbation theory following Dermine et al. (2013), the author argued that, a disk with a fine-tuned lifetime as long as $\sim 10^{5}$ yr and mass around a few $10^{-4} M_{\odot}$ may result in eccentricities of $e \simeq 0.01-0.15$ for orbital periods between 15 and 50 days.

Adopting the idea that the orbital eccentricity is caused by sudden mass loss, here we suggest another possible scenario to this problem. During the recycling phase the NS accretes mass and angular momentum from the donor star. When its central density goes up to the critical density of quark deconfinement, a phase transition from a NS to a strange quark star (SS) may take place within a short time, and the loss of binding energy of the NS, if not big enough to disrupt the binary, can induce an eccentricity of the orbit. If the phase transition occurs at the end of or after the recycling process, the SS appears as an eccentric binary MSP with a He WD companion. 


\section{The NS-SS transition scenario}

Below we describe in some detail how the the NS-SS transition can influence the formation of various types of MSPs.

The concept of SSs has been suggested decades ago (Itoh 1970; Bodmer 1971; Witten 1984; Farhi \& Jaffe 1984; Haensel et al. 1986; Alcock et al. 1986), and it was suggested that, due to the absolute stability of quark matter, at least some of the pulsars could be SSs. Phase transition occurs when the central density of a NS rises above the critical density for dissolution of baryons into their quark constituents. The detailed processes of quark deconfinement in massive NSs are not precisely known. It might be gradual, lasting around $10^{8}$ yr (Olinto 1987; Horvath \& Benvenuto 1988), or in a detonation mode, accompanied with energy released compatible with a core collapse supernova (e.g., Cheng \& Dai 1996; Ouved et al. 2002). Based on the standard equation of state (EOS) of neutron-rich matter, Staff et al. (2006) pointed out that the critical density of quark deconfinement is $\sim 5 \rho_{0}$, where $\rho_{0} \sim 2.7 \times 10^{14} \mathrm{~g} \mathrm{~cm}^{-3}$ is the nuclear saturation density. Although different EOSs give different masses when the critical density is reached, a gravitational mass of $1.8 M_{\odot}$ may be a reasonable estimate for a NS with a core of quark matter (Akmal et al. 1998).

Quite a few specific SS candidates (e.g., SAX J1808.8-3658, Li et al. 1999) have been proposed (see Weber 2005, for a review), usually based on their observational features in X-rays. The formation of a SS can also impact the dynamical evolution of the host binary, which is seldom mentioned in the literature. We note that efficient mass growth of NSs is most likely to take place in $\mathrm{LMXB}_{2}$, and the phase transition may occur in the following two situations.

In the first one, an accreting NS converts to be a SS during the LMXB stage when it becomes sufficiently massive. Some baryonic mass is abruptly transformed into its binding energy during the phase transition, and the orbit expands and becomes eccentric. The donor star is then detached from its Roche-lobe, temporarily halting the mass transfer, and the SS becomes a MSP. If the orbit is close enough, its high-energy radiation and particles might be able to ablate the companion star, leading to the formation of redbacks (Roberts 2013, for a review). Otherwise, after some time the companion star evolves to refill its Roche lobe and resumes the mass transfer. The orbit is then invariably circularized by tidal torques, so the binary evolves as a normal LMXB, producing a circular MSP/He WD binary.

\footnotetext{
${ }^{2} \mathrm{NSs}$ in intermediate-mass X-ray binaries usually accretes at a super-Eddington rate, so that most of the mass may be ejected. During the subsequent LMXB evolution when the accretion rate becomes subEddington, the NSs can keep the accreted material and grow in mass.
} 
In the second, if the NS's spin is accelerated to be too fast, the central density might be centrifugally diluted and lie below the critical density for pure quark matter. Phase transition is delayed, and takes place during the terminal stages of the mass transfer or even the subsequent MSP phase, during which the NS is spun down due to the propeller mechanism (Tauris 2012), or magnetic dipole and/or gravitational radiation, respectively. The binary will keep its eccentricity because of the extremely long circularization time for two compact component stars. That may be responsible for the the eccentric orbits of PSRs $\mathrm{J} 2234+06$ and J1946+3417.

\section{Phase transition-induced orbital change}

Similar as the Freire \& Tauris (2014) model, here the orbital change in the MSP binaries is assumed to be due to instantaneous mass loss. To find out the possible parameter space of the WD mass and the mass loss during the phase transition for PSRs J2234+06 and J1946+3417 from the MSP mass, the orbital period, and the eccentricity, we use a Monte Carlo method to simulate the orbital change in the following steps.

1. We assume that the current mass $M_{\mathrm{SS}}$ of the MSP is randomly distributed in the range of $1.4-2.0 M_{\odot}$ (Gangopadhyay et al. 2013). The upper limit is set in accord with recent measurements of two massive pulsars, PSR J1614-2230 of mass $1.97( \pm 0.04) M_{\odot}$ (Demorest et al. 2010) and PSR J0348+0432 of mass 2.01 $( \pm 0.04) M_{\odot}$ (Antoniadis et al. 2013).

2. The current mass $M_{\mathrm{WD}}$ of the He WD is then calculated from $M_{\mathrm{SS}}$ and the observed mass function $M_{\mathrm{WD}}^{3} \sin ^{3} i /\left(M_{\mathrm{SS}}+M_{\mathrm{WD}}\right)^{2}=0.0027$ for PSR J2234+06 (Deneva et al. 2013), where $i$ is the inclination angle of the orbit. We assume that $\cos i$ is uniformly distributed between 0 and 1.

3. Assuming that the binary orbit is initially circular and the current eccentricity (assumed to be in the range of $0.11-0.15$ ) is induced by instantaneous gravitational mass loss of the $\mathrm{NS}$, we can get the amount of the mass loss $\Delta M=e \cdot\left(M_{\mathrm{WD}}+M_{\mathrm{SS}}\right)$ and the gravitational mass $M_{\mathrm{NS}}\left(=M_{\mathrm{SS}}+\Delta M\right)$ of the NS (Bhattacharya \& van den Heuvel 1991), if the energy releasing during the phase transition is spherically symmetric and there is no kick imparted to the SS.

4. Not all of the obtained values of $\Delta M$ and $M_{\mathrm{WD}}$ are physically reasonable, and we select those which satisfy the following condition predicted by LMXB evolution. On one hand, from $M_{\mathrm{SS}}, M_{\mathrm{WD}}$ and the current orbital period (assumed to be in the range of $22-32$ days), we get the orbital separation $a$. Combining $a$ and the eccentricity $e$ yields the separation $a_{\mathrm{i}}$ 
before the phase transition via the equation $a_{\mathrm{i}}=a(1-e)$. On the other hand, we derive the orbital period $P_{\text {orb, i }}$ before the phase transition from $M_{\mathrm{WD}}$, using the orbital period - WD

mass relation given by Tauris \& Savonije (1999) for solar metallicities $(Z=0.02)$. Then from $P_{\mathrm{orb}, \mathrm{i}}, M_{\mathrm{NS}}$, and $M_{\mathrm{WD}}$ we also obtain $a_{\mathrm{i}}$ from the Kepler's law. We require that the value of this $a_{\mathrm{i}}$ should match the former one within $1 \%$.

The simulated $\Delta M$ vs. $M_{\mathrm{WD}}$ distribution is shown in Fig. 1. The allowed maximum NS mass is around $2.3 M_{\odot}$. It is seen that the mass loss is about $0.20-0.34 M_{\odot}$, and the WD mass is constrained to be about $0.257-0.275 M_{\odot}$ (red dots). If we decrease $e$ down to 0.027 (the eccentricity of PSR J1618-3919, which might have the same formation mechanism), the mass loss can be as low as about $0.05 M_{\odot}$, while the upper limit of the possible WD mass increases to about $0.278 M_{\odot}$ (blue dots). Simulation with the mass function $=0.0039$ for PSR J1946+3417 (Barr er al. 2013) gives similar result, as shown in Fig. 2. These numbers are compatible with the standard evolution of LMXBs.

The observed range of the orbital periods for eccentric MSPs thus far is $22-32$ days, and the corresponding orbital periods before phase transition are found to range from 15 to 35 days. It is not clear why phase transition would only occur for such a particular range of orbital periods. It might be related to the requirement that phase transition (supposedly in a massive NS) should occur at (or near) the end of mass transfer, in order to produce observable eccentric MSPs. According to the studies of Li \& Wang (1998) and Tauris \& Savonije (1999) (see also Zhu et al. 2013), the amount of mass accreted by a NS during the LMXB evolution generally decreases with the orbital period, probably due to the thermal-viscous instability in the accretion disks (Lasota 2001). In systems with longer orbital periods, the NS may not accrete sufficient mass to trigger phase transition, while NSs in narrower orbits may get enough material and undergo phase transition during the LMXB phase, but the resulting eccentric orbits would be circularized by the subsequent mass transfer. So we tentatively speculate that only LMXBs with a particular range of orbital periods may lead to the formation of eccentric MSPs. However, we need to caution that the accretion processes in LMXBs are rather complicated. Considering the fact the mass transfer is very likely to be nonconservative and the big uncertainties in the accretion efficiency, it is premature to accurately estimate the accreted mass by a NS.

\section{Discussion}

Based on the assumption that massive NSs may convert to be SSs, we suggest that the sudden loss of gravitational mass during the phase transition may account for the eccentric orbits in the two recycled binary pulsars, which are otherwise thought to be in circular orbits. 
Recent population synthesis calculation by Zhu et al. (2013) suggested that about $0.1-$ $10 \%$ of LMXBs can produce SSs, depending on the masses of the nascent NSs and the fraction of transferred matter accreted by the NSs. Observational clues have also been proposed for the existence of SSs. For example, it is known that special global oscillation modes (r-modes) that emit gravitational waves would prohibit pulsars from spinning with high frequencies, unless the damping of these modes, determined by the microscopic properties of matter, can prevent this (e.g., Andersson \& Kokkotas 2001). Alford \& Schwenzer (2014) showed that for each form of matter there is a distinct region in the spin frequency/spindown-rate diagram where r-modes can be present. They found that stars containing ungapped quark matter are consistent with both the observed radio and X-ray data, whereas, even when taking into account the considerable uncertainties, NS models with standard viscous damping are inconsistent with both data sets and additional damping mechanisms would be required.

Our phase transition scenario can be compared with others in the following aspects, which are to be tested by future observations.

1. Although phase transitions are expected to occur in massive NSs, the masses of the resultant SS MSPs could lie in a wide range (Gangopadhyay et al. 2013), depending on the amount of the mass that is transferred into the binding energy. This is similar to the Antoniadis (2014) prediction but different from the simplest scenario in Freire \& Tauris (2014) (that of a rigidly rotating WD before the collapse), which predicts that these MSPs should be exceptionally light.

2. The eccentric MSPs probably have a He WD companion with mass in a relatively narrow range $\left(\sim 0.25-0.28 M_{\odot}\right)$. With such WD companions, the NSs are likely to be heavy at the moment of phase transition, implying that the resultant SS pulsars are fully recycled pulsars.

3. The eccentric MSPs may have kinematic properties (i.e., Galactic heights and peculiar velocities) at least similar as what is observed in the normal MSP population, since the supernova that formed the NS should give the system a reasonable kick. This is again similar to the Antoniadis (2014) prediction but contrasts with that of Freire \& Tauris (2014). In the latter scenario, the AIC is the only supernova that ever occurs and, because this supernova is so symmetric, the eccentric systems generated would have very small peculiar velocities and Galactic heights.

As noted before, phase transition may also occur during the LMXB phase. The conversion of a NS into a SS could lead to a quark nova (Ouyed et al. 2002, 2011), with energy released comparable to a normal supernova. A peculiar X-ray binary, Cir X-1, might be produced in this way. This source has an orbital period of 16.6 days (Kaluzienski et al. 1976). It 
does not seem to fit either a high-mass or a low-mass X-ray binary. Detection of type-I X-ray bursts (Tennant et al. 1986; Linares et al. 2010) firmly establishes its nature as a low-field $\left(<10^{10} \mathrm{G}\right)$ NS, similar as those in LMXBs. The observed spectral features (Shirev et al. 1999; Soleri et al. 2009) and kilo-Hertz quasi-periodic oscillations (Boutloukos et al. 2006) also lend strong support to this conjecture. However, the orbit is shown to be eccentric with $e=0.45$ (Jonker et al. 2007), suggesting that the binary was recently subject to mass loss (and/or a kick) during the formation of the compact star. Moreover, Heinz et al. (2013) discovered a supernova remnant (with age less than 4600 yr) surrounding Cir X-1, making it the youngest known X-ray binary. Jonker et al. (2007) associated the observed optical absorption-line spectrum with the companion star of Cir X-1, suggesting that the companion star could be a B5-A0 supergiant. However, the possibility cannot be excluded that the spectrum originates in the accretion disk, and in that scenario, the companion star is likely to be a (sub)giant of mass around $0.4 M_{\odot}$. If it is the case the conflicting properties (young age, eccentric orbit, and low magnetic field) of Cir X-1 can be well accounted for by the NS-SS phase transition scenario. The key point is that the supernova remnant and the eccentric orbits were produced by the quark nova when the accreting NS was converted to be a SS, rather than the supernova that originally formed the NS.

No matter if our specific scenario is confirmed or ruled out by future observations, the present data discussed in this paper will provide useful constraints on the formation channels of these peculiar systems, and there will be deep consequences for the physics of strong interactions.

We are grateful to an anonymous referee for helpful and constructive comments. This work was supported by the Natural Science Foundation of China under grant Nos. 11133001 and 11333004, and the Strategic Priority Research Program of CAS under grant No. XDB09010000.

\section{REFERENCES}

Akmal, A., Pandharipande, V. R., \& Ravenhall, D. G. 1998, Phys. Rev. C, 58, 1804

Alcock, C., Farhi, E., \& Olinto, A. V. 1986, ApJ, 310, 261

Alexander, D. R., \& Ferguson, J. W. 1994, ApJ, 437, 879

Alford, M. \& Schwenzer, K. 2014, Phys. Rev. Lett., 113, 251102

Alpar, A., Cheng, A. F., Ruderman, M. A., \& Shaham, J. 1982, Nature, 300, 728 
Andersson, N., \& Kokkotas, K. D. 2001, Int. J. Mod. Phys. D, 10, 381

Antoniadis, J. 2014, ApJ, 797, L24

Antoniadis, J., Freire, P. C. C., Wex, N., et al. 2013, Science, 340, 1233232

Archibald, A. M., Stairs, I. H., Ransom, S. M., et al. 2009, Science, 324, 1411

Bailes, M. 2010, New A Rev., 54, 80

Barr, E. D., Champion, D. J., Kramer, M., et al., 2013, MNRAS, 435, 2234

Bhattacharya, D., \& van den Heuvel, E. P. J. 1991, Phys. Rep., 203, 1

Bodmer, A. R. 1971, Phys. Rev. D, 4, 1601

Boutloukos, S., van der Klis, M., Altamirano, D. et al. 2006, ApJ, 653, 1435

Champion, D. J., Ransom, S. M., Lazarus, P., et al. 2008, Science, 320, 1309

Cheng, K. S., \& Dai, Z. G. 1996, Phys. Rev. Lett., 77, 1210

Demorest, P. B., Pennucci, T., Ransom, S. M., et al. 2010, Nature, 467, 1081

Deneva, J. S., Stovall, K., McLaughlin, M. A., et al., 2013, ApJ, 775, 51

Dermine, T., Izzard, R. G., Jorissen, A., \& Van Winckel, H. 2013, A\&A, 551, A50

Farhi, E., \& Jaffe, R. L. 1984, Phys. Rev. D, 30, 2379

Freire, P. C. C., \& Tauris, T. M. 2014, MNRAS, 438, 86

Freire, P. C. C., Verbiest, J. P. W., Tauris, T. M. et al. 2011, MNRAS, 412, 2763

Gangopadhyay, T., Ray, S., Li, X.-D., Dey, J., \& Dey, M. 2013, MNRAS, 431, 3216

Haensel, P., Zedunik, J. L., \& Schaeffer, R. 1986, A\&A, 160, 121

Heinz, S., Sell, P., Fender, R. P., et al. 2013, ApJ, 779, 171

Horvath, J. E., \& Benvenuto, O. G. 1988, Phys. Lett. B, 213, 516

Hut, P. 1981, A\&A, 99, 126

Itoh, N. 1970, Prog. Thor. Phys., 44, 291

Jonker, P. G., Nelemans, G., \& Bassa, C. G. 2007, MNRAS, 374, 999 
Kaluzienski, L. J., Holt, S. S., Boldt, E. A., \& Serlemitsos, P. J. 1976, ApJ, 208, L71

Knispel, B., Lyne, A. G., Stappers, B. W., et al. 2015, arXiv: 1504.03684

Lasota, J.-P. 2001, New A Rev., 45, 449

Li, X.-D., Bombaci, I., Dey, M., Dey, J., \& van den Heuvel, E. P. J. 1999, Phys. Rev. Lett., 83,3776

Li, X.-D., \& Wang, Z.-R. 1998, ApJ, 500, 935

Linares, M., Watts, A., Altamirano, D., et al. 2010, ApJ, 719, L84

Liu, X.-W., \& Li, X.-D. 2009, ApJ, 692, 723

Lorimer, D. R. 2008, Living Rev. Relativity, 11, 8

Olinto, A. V. 1987, Phys. Lett. B, 192, 71

Papitto, A., Ferrigno, C., Bozzo, E., et al. 2013, Nature, 501, 517

Phinney, E. S. 1992, Phil. Trans. Phys. Sc. \& Eng., 341, 39

Pijloo, J. T., Caputo, D. P., \& Portegies Zwart, S. F. 2012, MNRAS, 424, 2914

Pols, O. R., Tout, C. A., Eggleton, P. P., \& Han, Z. 1995, MNRAS, 274, 964

Portegies Zwart, S., van den Heuvel, E. P. J., van Leeuwen, J., \& Nelemans, G. 2011, ApJ, 734,55

Pylyser, E., \& Savonije, G. J. 1989, A\&A, 208, 52

Ouyed, R., Dey, J., \& Dey, M. 2002, A\&A, 390, L39

Ouyed, R., Staff, J., \& Jaikumar, P. 2011, ApJ, 729, 60

Radhakrishnan, V., \& Srinivasan, G. 1982, Current Science, 51, 1096

Roberts, M. S. E. 2013, in IAU Symp., 291, Neutron Stars and Pulsars: Challenges and Opportunities After 80 Years, ed. J. van Leeuwen (Cambridge: Cambridge Univ. Press), 127

Roy, J., Ray, P. S., Bhattacharyya, B. et al. 2015, ApJ, 800, L12

Shirey, R. E., Bradt, H. V., \& Levine, A. M. 1999, ApJ, 517, 472 
Soleri, P., Tudose, V., Fender, R., van der Klis, M., \& Jonker, P. G. 2009, MNRAS, 399, 453

Staff, J. E., Ouyed, R., \& Jaikumar, P. 2006, ApJ, 645, L145

Stairs, I. H., Faulkner, A. J., Lyne, A. G., et al. 2005, ApJ, 632, 1060

Tauris, T. M. 2012, Science, 335, 561

Tauris, T. M., Sanyal, D., Yoon, S.-C. \& Langer, N. 2013, A\&A, 558, A39

Tauris, T. M., \& Savonije, G. J. 1999, A\&A, 350, 928

Tennant, A. F., Fabian, A. C., \& Shafer, R. A. 1986, MNRAS, 221, 27

Weber, F. 2005, Prog. Part. \& Nucl. Phys., 54, 193

Witten, E. 1984, Phys. Rev. D, 30, 272

Yaron, O., Prialnik, D., Shara, M. M., \& Kovetz, A. 2005, ApJ, 623, 398

Yoon, S.-C., \& Langer, N. 2004, A\&A, 419, 623

Zahn, J.-P. 1977, A\&A, 57, 383

Zhu, C., Lü, G., Wang, Z., \& Liu, J. 2013, PASP, 125, 25 


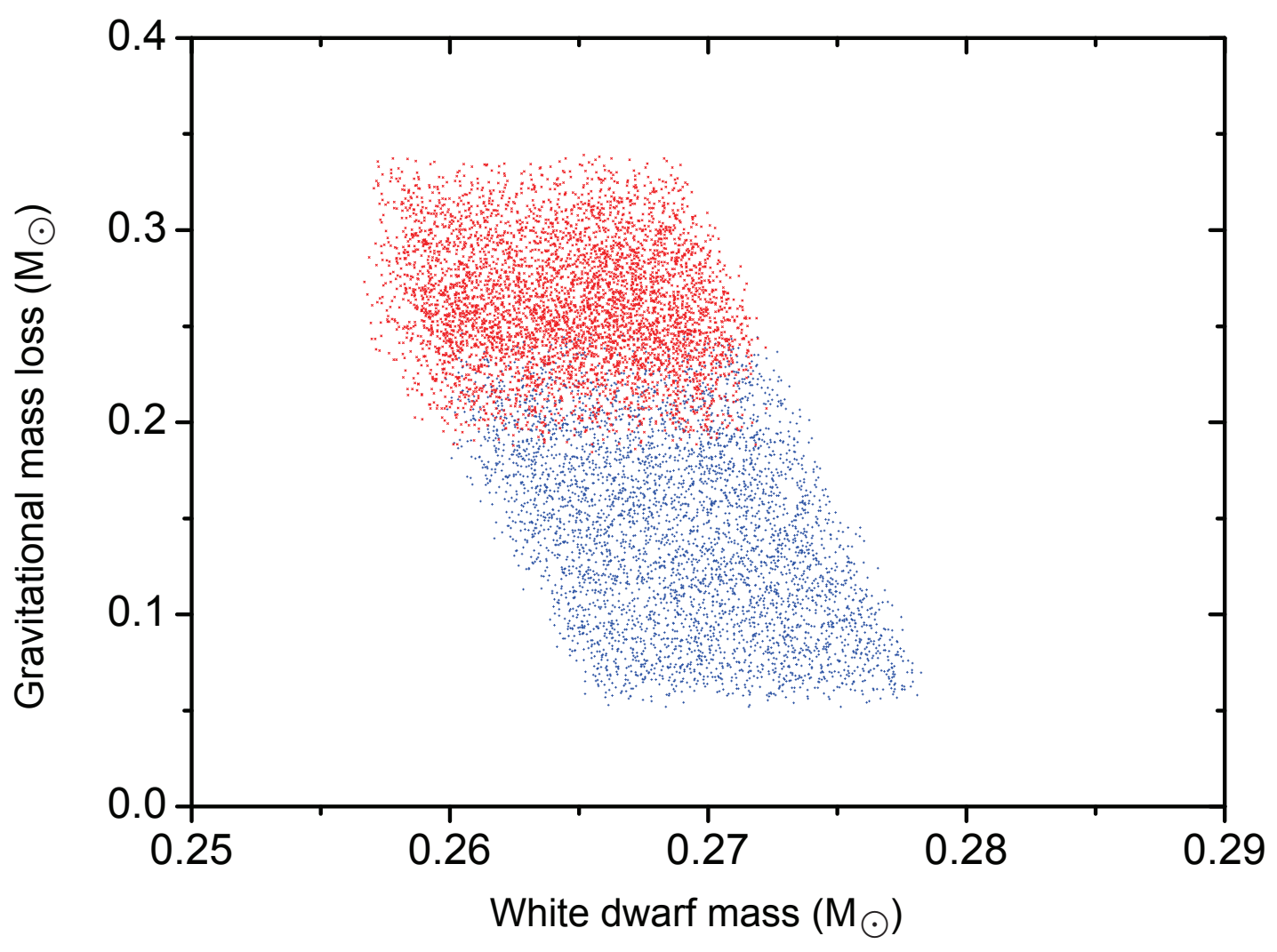

Fig. 1.- Required gravitational mass loss of the NS vs. the WD mass in the phase transition scenario to reproduce the properties of PSR J2234+06. The red and blue dots represent the cases for the eccentricity in the range of $0.11-0.15$ and $0.027-0.11$, respectively. 


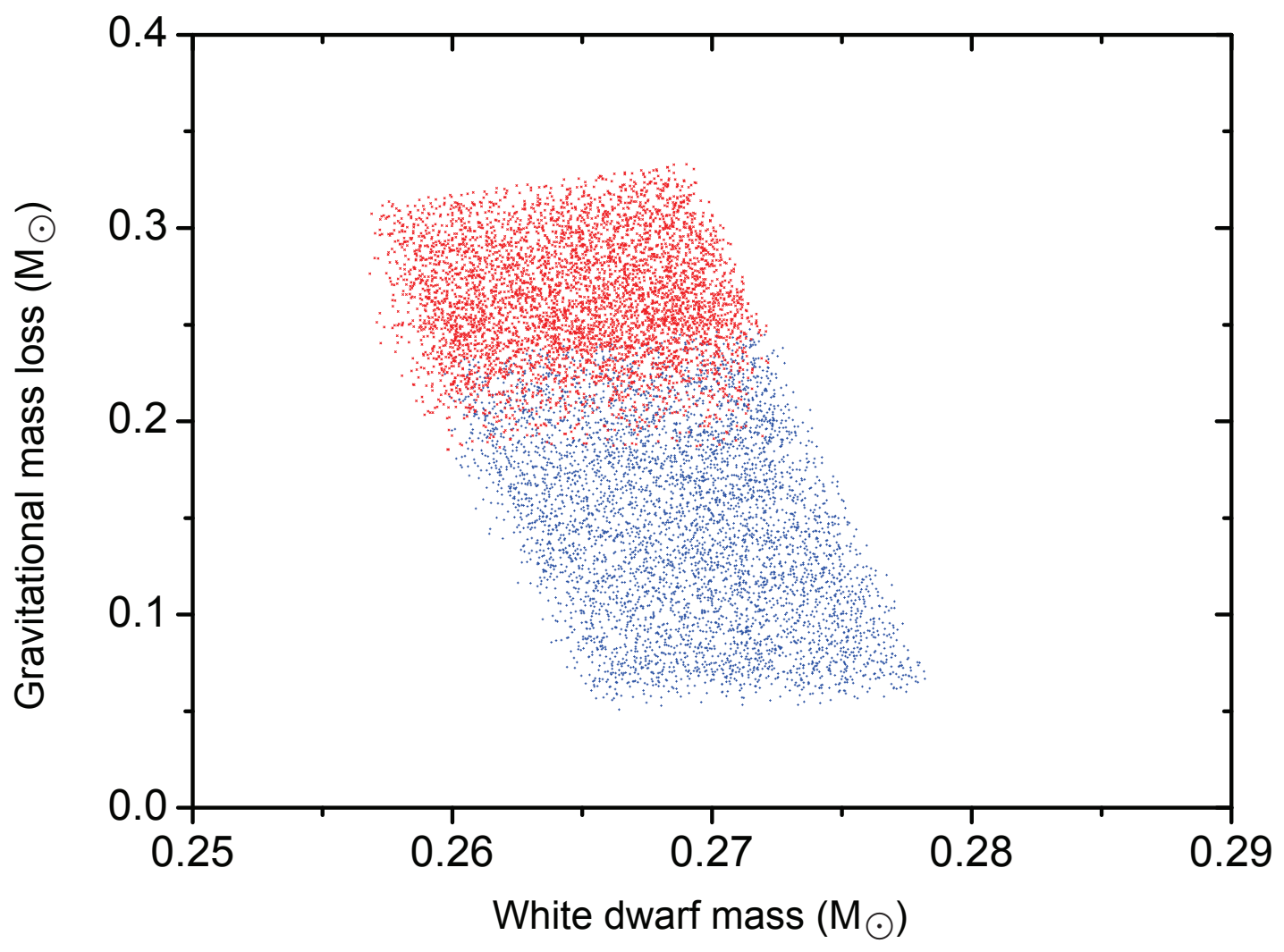

Fig. 2.- Same as Fig. 1 but with the mass function for PSR J1946+3417. 